\title{
Development and Fabrication of Advanced Materials for Energy and Environment Applications
}

\author{
Wen Zeng, ${ }^{1}$ Ming-Guo $\mathrm{Ma}^{2}$ Jie-Fang $\mathrm{Zhu}^{3}$ and Shao-Wen $\mathrm{Cao}^{4}$ \\ ${ }^{1}$ College of Materials Science and Engineering, Chongqing University, Chongqing 400040, China \\ ${ }^{2}$ Beijing Key Laboratory of Lignocellulosic Chemistry, College of Materials Science and Technology, Beijing Forestry University, \\ Beijing 100083, China \\ ${ }^{3}$ Department of Materials Chemistry, The Ångström Laboratory, Uppsala University, 75121 Uppsala, Sweden \\ ${ }^{4}$ School of Materials Science and Engineering, Nanyang Technological University, Singapore 639798
}

Correspondence should be addressed to Wen Zeng; wenzeng@cqu.edu.cn and Ming-Guo Ma; mg_ma@bjfu.edu.cn

Received 25 July 2013; Accepted 25 July 2013

Copyright (C) 2013 Wen Zeng et al. This is an open access article distributed under the Creative Commons Attribution License, which permits unrestricted use, distribution, and reproduction in any medium, provided the original work is properly cited.

The increasing environmental problems and deficiencies of sustainable energy sources have become serious issues that are urgently in need of being addressed. To make up this issue, the key is of development and fabrication of advanced materials that for energy and environment applications.

The performance of materials depends critically on their microstructures, which requires the development of materials processing techniques to obtain the desired microstructures and morphologies. It is of great importance in future technological applications to understand how the materials behave at nanoscale during working and how controllably manufacture. No matter physical or chemical preparation, forwardlooking theoretical guidance and characterization proof are necessary for explaining the formation mechanism so as to design devices with expected properties for nonpolluting energy generation and environmental protection. In this issue, different approaches were used to create nanostructures in several oxide materials dealing with the environmental and energy-converting problems.

The paper "Pd-doped $\mathrm{SnO}_{2}$-based sensor detecting hydrocarbon characteristic fault/hydrocarbon gases in transformer" by W. Chen et al. synthesized Pd-doped $\mathrm{SnO}_{2}$ nanoparticles and measured their gas-sensing properties towards methane, ethane, ethylene, and acetylene. The sensing mechanism was also addressed in detail based on a quantum chemistry calculation of molecule orbital energy. The paper "Application of flower-like $\mathrm{ZnO}$ nanorods gas sensor detecting $\mathrm{SF}_{6}$ decomposition products" by S. Peng et al. proposes a novel gas sensor based on flower-like $\mathrm{ZnO}$ nanorods to detect typical $\mathrm{SF}_{6}$ decompositions. This sensor shows a remarkable discrimination among $\mathrm{SO}_{2}, \mathrm{SOF}_{2}$, and $\mathrm{SO}_{2} \mathrm{~F}_{2}$ with high linearity. The paper "Gas sensing properties and mechanism of nano$\mathrm{SnO}_{2}$ based sensor for hydrogen and carbon monoxide" by W. $\mathrm{Chen}$ et al. prepared nano- $\mathrm{SnO}_{2}$ powder via a hydrothermal method and investigated the gas sensing performances of the obtained sensor against power transformer fault gases such as hydrogen and carbon monoxide. The gas sensing mechanism was qualitatively explained by the first-principles calculation. These results will present a new feasible way on exploring new metal oxide gas sensor for on-line monitoring of fault gases dissolved in power transformers.

The paper "HER catalytic activity of electrodeposited Ni$P$ nanowires under the influence of magnetic field" by H.-B. Lee et al. fabricated the nickel alloy electrodes both in plane and nanowires morphologies by electrodepositing in sulfamate bath. By using nanowires morphology and applying in magnetic field, the HER stability of the Ni electrode increased in more than four folds than that of its plane counterpart.

The paper "The development of photocatalyst with hybrid material $\mathrm{CNT} / \mathrm{TiO} \mathrm{O}_{2}$ thin films for dye-sensitized solar cell" by Y. W. Kim and S. H. Park prepared $\mathrm{CNT} / \mathrm{TiO}_{2}$ as a hybrid material to improve their efficiency of dye-sensitized solar cell. The higher concentration of CNT will decrease the transmission and resistance of $\mathrm{TiO}_{2}$ thin film. 
The paper "Fabrication and characterization of manganese ferrite nanospheres as a magnetic adsorbent of chromium" L.-X. Yang et al. synthesized the manganese ferrite nanospheres by a one-step and template-free solvothermal method. The obtained materials show an excellent ability to magnetic removal of chromium in waste water.

The paper "Preparation of smooth surface $\mathrm{TiO}_{2}$ photoanode for high energy conversion efficiency in dye-sensitized solar cells" S. Kathirvel et al. fabricated the dye-sensitized solar cells by using a $\mathrm{TiO}_{2}$ nanosol-based photoelectrode. These cells show an overall light conversion efficiency and a short-circuit current density.

The paper "Effect of solution spray rate on the properties of chemically sprayed ZnO:In thin films" by M. Kriisa et al. using a chemical spray method to prepare $\mathrm{ZnO}$ :In thin films on glass substrates and found that the solution spray rate is an important technological parameter controlling the film thickness and electrical properties of the sprayed $\mathrm{ZnO}$ :In films.

The paper "Influence of hydrothermal temperature on phosphorus recovery efficiency of porous calcium silicate hydrate" by W. Guan et al. synthesized porous calcium silicate hydrate by carbide residue and white carbon black. The influence of hydrothermal temperature on phosphorus recovery efficiency was investigated in detail.

The paper "Preparation of baking-free brick from manganese residue and its mechanical properties" by P. Wang and D.-y. liu addressed a summarization of the main methods to produce building materials from manganese slag materials. It is concluded that the baking-free brick prepared from manganese residue could have excellent compressive strength performance under certain formula.

The paper "Characterization of nanoporous ceramic granules made with coal fly ash and their utilization in phenol removal from water" by $Z$. Jing Jing used the coal fly ash to produce composite ceramic granules with high porosity and specific surface area. Effects of various factors such as adsorbents dosage, contact time, and temperature were investigated and aimed to obtain optimized parameter.

The paper "Facile synthesis of Fe-doped titanate nanotubes with enhanced photocatalytic activity for castor oil oxidation" by $\mathrm{G}$. Fu et al. reported the synthesis of the iron doped $\mathrm{Ti}$ nanotubes and provided a facile route to improve the photocatalytic efficiency of materials and other properties such as magnetic property.

The paper "Mineral phase and physical properties of red mud calcined at different temperatures" by C.-S. Wu and D.-y.Liu investigated the phase composition and structural transition of red mud from room temperature to $1200^{\circ} \mathrm{C}$. The obtained results provided an important base for the further studies of comprehensive utilization of red mud.

The paper "Synthesis of octahedral-shaped $\mathrm{NiO}$ and approaches to an anode material of manufactured solid oxide fuel cells using the decalcomania method" by $\mathrm{H}$. Cho et al. synthesized octahedral-shaped $\mathrm{NiO}$ particles by microwave thermal method. Using an octahedral $\mathrm{NiO}$ anode functional layer, fuel cell performance was significantly improved owing to better contact between the electrolyte and anode.
The paper "Characterization of porous $\mathrm{WO}_{3}$ electrochromic device by electrochemical impedance spectroscopy" by C. C. Chen reported the microstructure of the anodic tungsten oxide and its use in an electrochromic glass device. The fabrication process can be extended to other metal oxides such as $\mathrm{TiO}_{2}$ and $\mathrm{Ta}_{2} \mathrm{O}_{5}$, which are electrochromic and solid charge conductive films.

By compiling these papers, we hope this issue will provide some valuable insights into our readers and researchers with respect to the field of advanced materials for energy and environment applications.
Wen Zeng Ming-Guo Ma Jie-Fang Zhu Shao-Wen Cao 

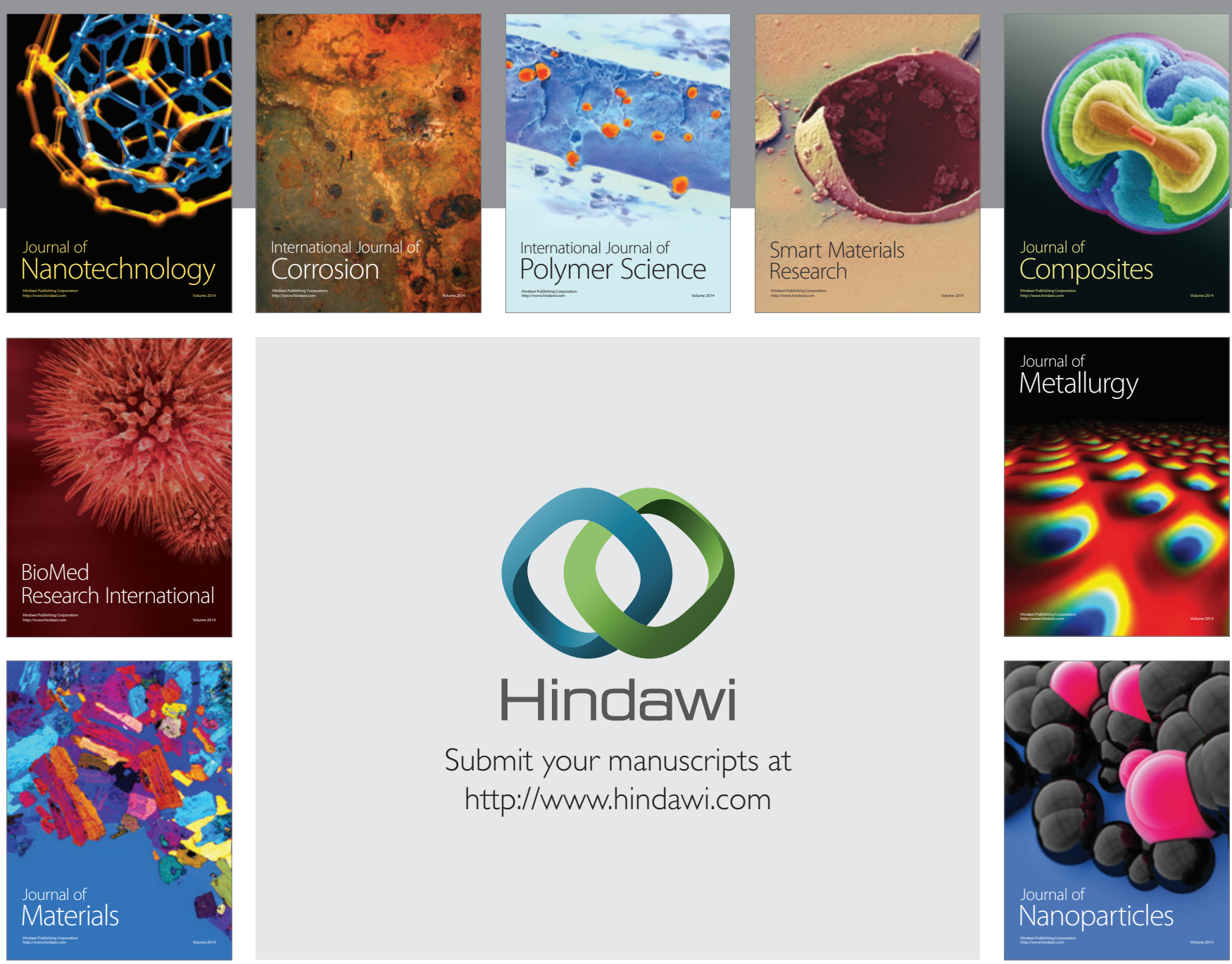

Submit your manuscripts at http://www.hindawi.com
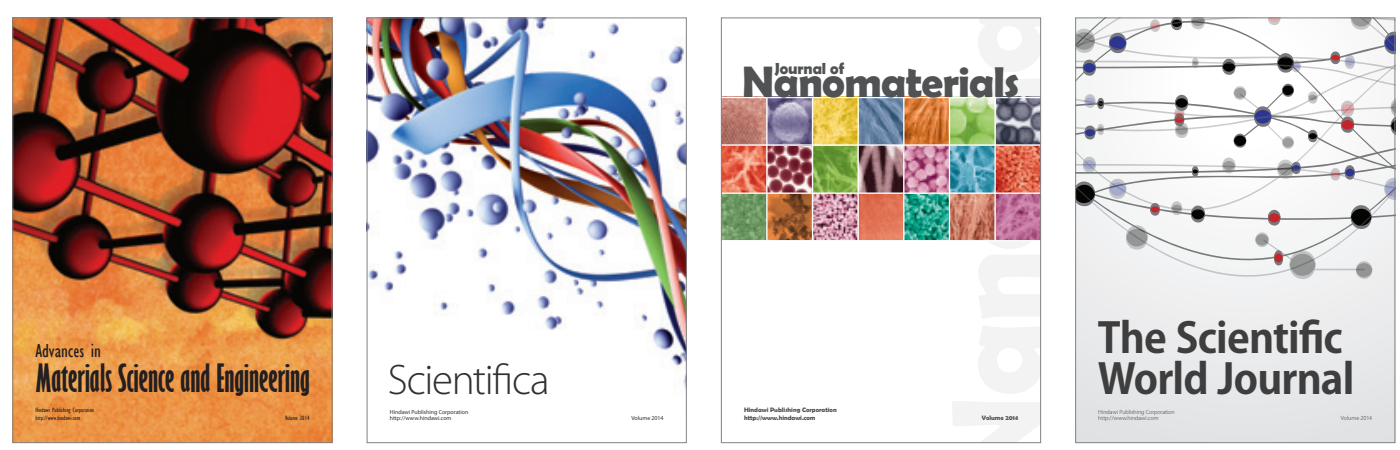

\section{The Scientific World Journal}
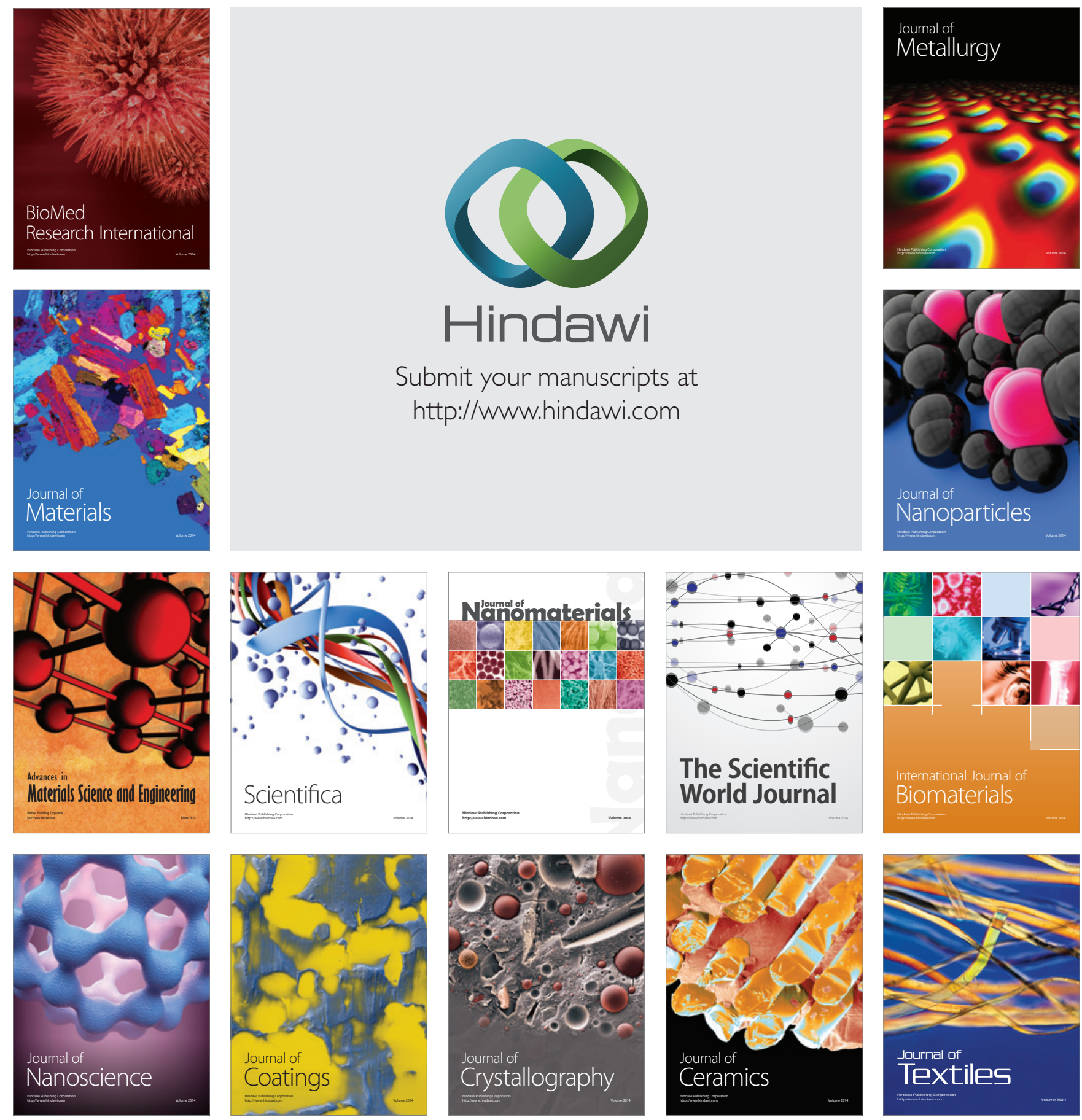